\title{
Renal artery stenosis and hypertension
}

\author{
Frans H M Derkx, Maarten A D H Schalekamp
}

If essential hypertension is a disease of theories, then renovascular hypertension is a disease of experiments. These experiments are to a large extent the basis for our diagnostic and therapeutic approach to the patient with renal artery stenosis. It is not always justifiable to label hypertension in the presence of renal artery stenosis as renovascular hypertension. The two conditions may simply coexist. Alternatively, it is even possible that the raised blood pressure is the cause of the stenosis and not the other way around-ie, hypertension increases the risk of atherosclerotic vascular disease, which may also involve the renal arteries.

Timely diagnosis of renovascular hypertension is important because this condition carries a worse prognosis than essential hypertension and seems to be less amenable to drug treatment, with greater risks of dose-dependent side-effects. Renovascular hypertension also carries a higher risk of progression to accelerated or malignant hypertension, and may result in irreversible ischaemic failure of the affected kidney. ${ }^{1,2}$

The prevalence of renovascular disease is less than $1 \%$ in the general population of hypertensives, $5 \%$ in hospitalbased populations, and up to $40 \%$ in patients referred to hypertension clinics. In two-thirds of cases the cause of renovascular hypertension is atherosclerotic disease; less common causes are fibromuscular dysplasia, arteritis, thrombosis, arterial dissection, and stenosis in a transplanted kidney.

\section{When to look for renal artery stenosis}

Renovascular hypertension is usually symptomless. Hypertension that is difficult to control medically is probably the best indication that further diagnostic evaluation is warranted. A clue is a rise in serum creatinine during treatment with an angiotensin converting enzyme (ACE) inhibitor. In an ongoing prospective randomised multicentre trial of percutaneous transluminal renal angioplasty (PTRA) vs medical treatment, we found that the prevalence of renal artery stenosis in a group of 80 drug-resistant hypertensive patients was almost $30 \%{ }^{3}$ Drug-resistance was defined as a diastolic pressure of 95 $\mathrm{mm} \mathrm{Hg}$ or above during three consecutive visits in patients randomised to either the combination of enalapril $20 \mathrm{mg}$ and hydrochlorothiazide $25 \mathrm{mg}$ or to amlodipine $10 \mathrm{mg}$ with atenolol $50 \mathrm{mg}$.

Diagnostic imaging tests are used to assess the location and severity of the stenotic lesions. Functional diagnostic

Department of Internal Medicine I, University Hospltal Dijkzigt, Erasmus University, Rotterdam, Netherlands (F H M Derkx MD, MAD H Schalekamp MD)

Correspondence to: Dr F H M Derkx, Department of Internal Medicine I, University Hospital Dijkzigt, Room L242,

Dr Molewaterplein 40, 3015 GD Rotterdam, Netherlands tests are carried out to ascertain the pathophysiological importance of the stenosis. Some functional tests can be used to predict cure or improvement of hypertension. A scheme for diagnosing renovascular hypertension is shown in the figure.

\section{Diagnostic imaging tests}

\section{Arteriography}

Contrast angiography is the only method for direct visualisation of the renal arteries and is the gold standard for comparison with less invasive procedures. Most clinicians judge narrowing of the arterial lumen of at least $50 \%$ to be significant, although perfusion pressure distal from the stenosis may be maintained until the narrowing exceeds $70 \%{ }^{4}$ The frequency of serious complications requiring medical support varies from 0.2 to $2.3 \% .^{5}$ Intra-arterial digital subtraction angiography with low-dose contrast medium usually gives excellent images. However, additional high-dose contrast arteriography may be necessary for more detailed information. Intravenous digital subtraction angiography is less invasive but requires more contrast medium, and the quality of the images is often unsatisfactory because renal branches are not adequately displayed. ${ }^{1}$

\section{Duplex doppler sonography and newer techniques}

Duplex doppler sonography combines traditional ultrasound imaging with a doppler technique to measure blood flow velocities in the renal arteries. This method is time-consuming and highly dependent on the operator. Even when done by experienced investigators as many as $15 \%$ of studies cannot be evaluated. If one excludes such failures, sensitivity ranges from 0.63 to 1.0 and specificity from 0.73 to 0.96 .6 Doppler sonography can be especially useful for anatomical evaluation of PTRA or surgical treatment. ${ }^{7}$ The first reports of magnetic resonance imaging and three-dimensional spiral computed tomography are promising. ${ }^{6}$ Their application outside specialised centres needs further evaluation.

\section{Functional dlagnostic tests}

Rapid sequence intravenous urography is no longer accepted for diagnostic testing because of its low sensitivity and specificity and the risk of nephropathy. ${ }^{1}$ However, it may be helpful if one suspects coexisting urinary tract abnormalities.

\section{ACE inhibitor/plasma renin tests}

The juxtaglomerular apparatus of the affected kidney responds to the decreased perfusion pressure with increased renin secretion. However, even under strictly standardised conditions, $50 \%$ of patients with renovascular 


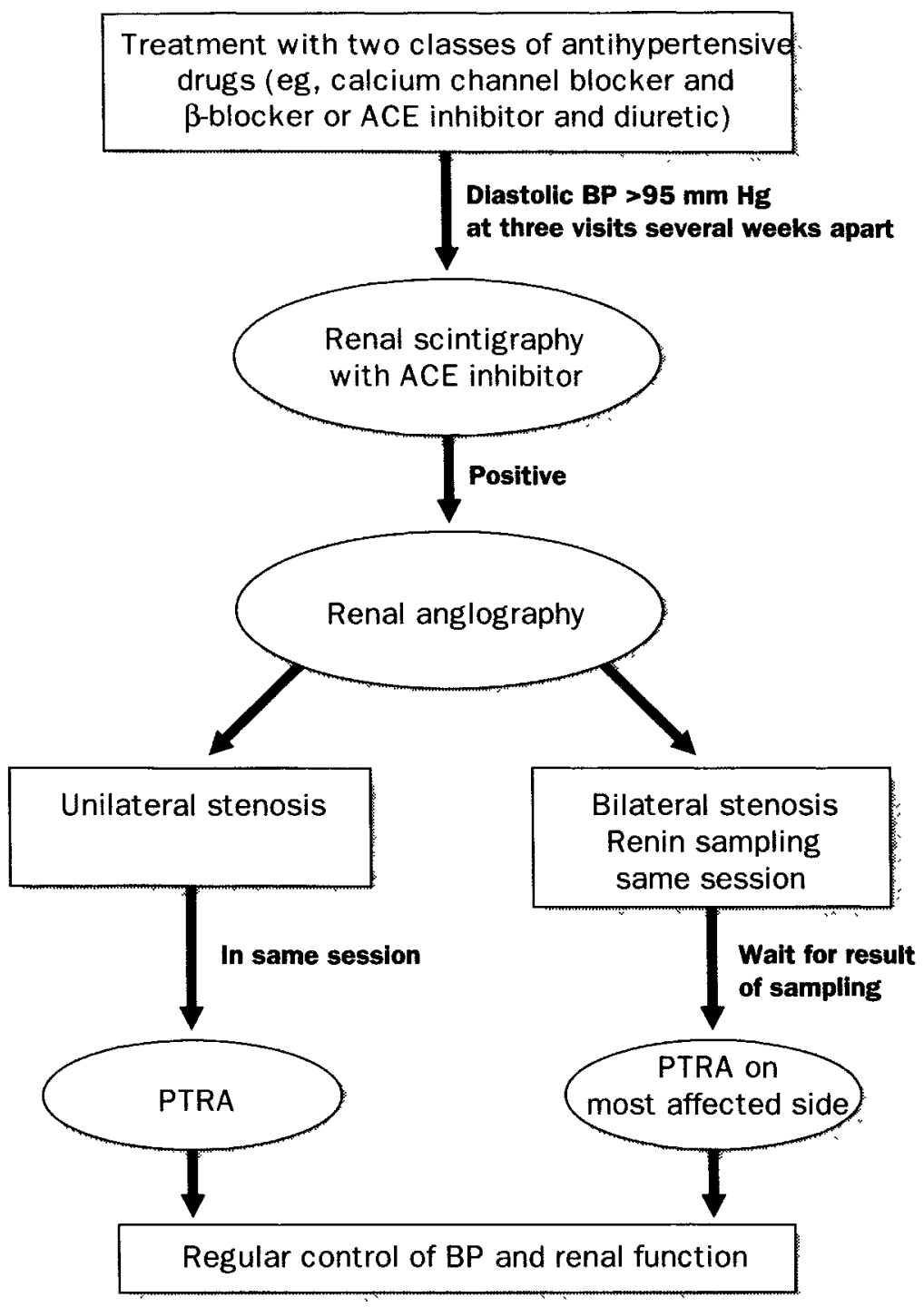

Figure: Evaluation of patients with renovascular disease

hypertension have peripheral vein plasma renin concentrations within the normal range. ${ }^{8}$ Curiously $10 \%$ of patients with essential hypertension likewise have raised plasma renin concentrations.

ACE inhibitors increase plasma renin by their hypotensive effect and by interrupting the feedback inhibition of renin release by angiotensin II. The captoprilrenin test is based on the exaggerated rise in peripheral vein renin that occurs in patients with renal artery stenosis after a single dose of captopril of 25 or $50 \mathrm{mg}$. Depending on the criteria for a positive test-ie, the stimulated concentration of renin or the absolute or percentage increment in renin-the sensitivity ranges from 0.73 to 1.0 in most reports. ${ }^{6}$ The specificity of this test ranges from 0.72 to 0.95 . To achieve acceptable sensitivity, the test has to be done under strictly standardised conditions that are often incompatible with outpatient evaluation.

\section{Renal scintigraphy with and without ACE inhibition}

After intravenous injection of $99 \mathrm{~m}$ Tc-labelled diethylenetriamine penta-acetic acid (DTPA) the kidney can be visualised and the contribution of each kidney to the total glomerular filtration rate can be estimated by external counting. ${ }^{9}$ Glomerular filtration rate on the affected side depends critically on angiotensin II. Consequently, ACE inhibition leads to impairment of renal function on the affected side, ${ }^{10}$ and this adverse effect of ACE inhibition can be turned to advantage because it increases the asymmetry between the affected and the non-affected kidney. Scintigraphy 1 hour after 25 or $50 \mathrm{mg}$ captopril is a valuable diagnostic test. ${ }^{11}$ Nevertheless, improvement in detection by the use of an ACE inhibitor is not a uniform finding. ${ }^{12,13}$
For outpatient evaluation, captopril-scintigraphy is more accurate than the captopril-renin test. ${ }^{13}$ Captoprilscintigraphy is also useful for detecting restenosis after PTRA or surgery.

99m Tc-labelled mercaptoacetyltriglycine (MAG3) may replace DPTA. ${ }^{9}$ Like hippurate, and unlike DTPA, MAG3 is secreted effectively by the proximal renal tubules. MAG3 scintigraphy gives good images even when renal function is impaired, but in patients with serum creatinine less than $200 \mu \mathrm{mol} / \mathrm{L}$ we have found that MAG3 is no better than DTPA for diagnosing renovascular hypertension.

\section{Tests to predlct outcome of PTRA or surgery}

\section{Renal vein renin measurements}

The renal production of renin equals the product of renal plasma flow and the veno-arterial difference in renin across the kidney. Blood sampling from the renal artery is unnecessary-peripheral blood can be used instead because arterial and peripheral venous renin concentrations are the same. The difference between the venous and arterial renin levels (V-A) is usually small by comparison with $V$ and $A$. The standard deviation of calculated $\mathrm{V}$-A difference equals the square root of $\left[\left(\mathrm{SD}_{\mathrm{V}}\right)^{2}+\left(\mathrm{SD}_{\mathrm{A}}\right)^{2}\right]$. This means that the $\mathrm{SD}_{\mathrm{V}-\mathrm{A}}$ is unacceptably high, since in renin assays the error is proportional to the assay result. Thus the $\mathrm{V} / \mathrm{A}$ ratio is used instead of the V-A difference. An important point is that an abnormally high $\mathrm{V} / \mathrm{A}$ ratio indicates a decrease in renal blood flow rather than an increase in renin production.

For conclusive results, sampling conditions during catheterisation must meet well-defined standards. ${ }^{14}$ Normally V/A averages $1 \cdot 25$. In about $90 \%$ of normal kidneys the ratio is below 1.50 . In $60-90 \%$ of the patients with unilateral renal artery stenosis the ratio on the affected side is 1.50 or more. In only $20 \%$ of normal kidneys is the ratio 1.10 or less, whereas in $50-80 \%$ of the patients with unilateral stenosis the ratio on the non-affected side is $1 \cdot 10$ or less. ${ }^{2,14,15}$

An increased V/A renin ratio on the affected side predicts a good outcome of PTRA or surgery, especially when the contralateral ratio is suppressed. ${ }^{14}$ The ratio between the venous concentrations on the affected and non-affected sides can also be used as a prognostic index. However, with this method it is impossible to show contralateral suppression, and the predictive value seems to be less than with the combined arterial and venous measurements. ${ }^{14}$

Some researchers have suggested that pretreatment with an ACE inhibitor may increase the predictive value of renal vein renin measurements. However, despite increased renin production, the renal vein-to-artery renin ratio, which is mainly related to renal blood flow, is not usually increased. ${ }^{15}$ Only when renin is measured shortly after ACE inhibition-eg, 15-45 min after captopril 25 or $50 \mathrm{mg}-$ when the arterial renin level is still rising, will the chance of finding an increased renal V/A ratio be improved. ${ }^{16} \mathrm{~A}$ disadvantage is the need for careful timing of captopril dosing, especially in patients already on ACE inhibitor treatment.

In $60-90 \%$ of patients with an increased non-stimulated $\mathrm{V} / \mathrm{A}$ renin ratio on the affected side, hypertension is cured or improved after PTRA or surgery, ${ }^{14}$ but about half the patients in whom the ratio is not increased likewise benefit from these interventions. Renal vein renin measurements therefore have limited value for predicting success of PTRA or surgery. Stimulation with captopril does not seem 
to improve the clinical usefulness of these measurements. Nevertheless, renal vein renin measurements may help clinicians to decide which side contributes most to the hypertension in patients with bilateral stenosis. ${ }^{17}$

\section{Renal scintigraphy with ACE inhibitor}

Results of a European multicentre trial indicated that an abnormal scintigram 1 hour after captopril 25 or $50 \mathrm{mg}$ is associated with cure or improvement of hypertension after PTRA or surgery. The sensitivity of this test was 0.95 and the specificity $0.82 .{ }^{11}$ Much lower figures have been reported by other groups.

\section{Treatment}

In many patients with renal artery stenosis blood pressure can be lowered by antihypertensive drugs. Concerns surrounding medical treatment are progression of the stenosis and harmful effects of blood pressure reduction on the function of the affected kidney. ${ }^{2}$ In a widely cited report published in $1974^{18}$ on patients with atherosclerotic renal artery stenosis, mortality over 7 to 14 years was $73 \%$ in patients who received drug treatment and $30 \%$ in those who underwent surgery ( 37 patients in each group). In a study started in 1971 and reported in $1981,{ }^{19} 4$ out of 41 patients with atherosclerotic renal artery stenosis who were randomly selected for drug therapy progressed to complete obstruction within 1 year. In 19 patients serum creatinine rose by $25-120 \%$. Thus, the results obtained with the drugs available before 1980 were disappointing. Whether PTRA or surgery is preferable to modern antihypertensive agents has not been established.

The main risks of PTRA are the same as those of arteriography. ${ }^{5}$ Complications specific for PTRA are dissection of the renal artery and cholesterol embolism. ${ }^{2}$ PTRA is the treatment of choice in patients with fibromuscular dysplasia ${ }^{20}$ and is now also widely used in atherosclerotic disease. A recent prospective randomised analysis of PTRA and surgery found no significant difference in outcome between the two approaches, which confirms the results of earlier non-randomised studies. ${ }^{20,21}$

The less invasive character of PTRA and its lower risk, especially in fragile elderly patients, favour PTRA over surgery. After successful PTRA, $20 \%$ of the patients are cured, $50 \%$ are improved, and $30 \%$ do not benefit. In $10-20 \%$ of patients PTRA is technically impossible.

In the randomised prospective study of PTRA vs surgery, ${ }^{21}$ restenosis was observed within 2 years in $25 \%$ of the patients after PTRA and in $4 \%$ after reconstructive surgery. In most patients restenosis can be cured by repeat PTRA.

There is some debate about whether ostial renal artery stenosis is a contraindication for PTRA, because of the high risk of restenosis. However, recent studies suggest that PTRA can be successful in such cases. ${ }^{22}$ Sometimes a balloon-expandable stainless steel stent may reduce the risk of stenosis after failure of a second PTRA; $;^{23}$ surgical revascularisation is another possibility.

\section{References}

1 Working group on renovascular hypertension. Detection, evaluation and treatment of renovascular hypertension. Arch Intern Med 1987 147: 820-29.

2 Pickering TG. Renovascular hypertension. Medical evaluation and non-surgical treatment. In: Laragh $\mathrm{JH}$, Brenner BM, eds.

Hypertension: pathophysiology, diagnosis, and management. New York: Raven, 1990: 1539 60.

3 van Jaarsveld BC, Derkx FHM, Man in 't Veld AJ, et al. Preliminary results of the Dutch Renal Artery Stenosis Intervention Cooperative (DRASTIC) study. Neth F Med 1994; 44: A65 (abstr).

4 May AG, van den Berg L, DeWeese JA. Hemodynamic effects of arterial stenosis. Surgery 1963; 53: 5213-24.

5 Neiman HL. Techniques of angiography. In: Neiman HL, Yao JS, eds. Angiography of vascular disease. New York: Churchill Livingstone, 1985: 1-27.

6 Davidson RA, Wilcox CS. Newer tests for the detection of the diagnosis of renovascular disease. $7 A M A$ 1992; 268: 3353-58.

7 Hudspeth DA, Hansen KJ, Reavis SW, Starr SM, Appel RG, Dean RH. Renal duplex sonography after treatment of renovascular disease. $\mathcal{F}$ Vasc Surg 1993; 18: 389-90.

8 Derkx FHM, Tan-Tjiong HI, Wenting GJ, Man in 't Veld AJ, Schalekamp MADH. Captopril test for diagnosing renal artery stenosis. In: Glorioso N, Laragh JH, Rapelli A, eds. Renovascular hypertension. New York: Raven, 1987: 295-304.

9 Blaufox MD, Fine EJ. Role of nuclear medicine techniques for evaluating hypertensive disease. In: Laragh JH, Brenner BM, eds. Hypertension: pathophysiology, diagnosis, and management. New York: Raven, 1990: 1509-34.

10 Wenting GJ, Tan-Tjiong HL, Derkx FHM, de Bruyn JHB, Man in ' $t$ Veld AJ, Schalekamp MADH. Split renal function after captopril in unilateral renal artery stenosis. BMF 1984; 288: 886-90.

11 Fommei E, Ghione S, Hilson AJW, et al. Captopril radionuclide test in renovascular hypertension: a European multicentre study. Eur $\mathcal{f} \mathrm{Nucl}$ Med 1993; 20: 617-23.

12 Derkx FHM, van Jaarsveld BC, Oei HY, et al. Does captopril improve the diagnostic value of ${ }^{99 \mathrm{~m}} \mathrm{TC}$-diethylenetriamine penta-acetic acid renography for renal artery stenosis? $\mathcal{F}$ Hypertens 1993; 11 (suppl 5): S200-01.

13 Elliott WJ, Martin WB, Murphy MB. Comparison of two noninvasive screening tests for renovascular hypertension. Arch Intern Med 1993; 153: 755-64.

14 Marks LS, Maxwell MH. Renal vein renin. Value and limitations in the prediction of operative results. Urol Clin N Am 1975; 2: 311-25.

15 . Derkx FHM, van den Meiracker AH, van Jaarsveld BC, et al. Captopril in the diagnostic work-up of patients with suspected renal artery stenosis. Neth $\mathcal{}$ Med 1993; 43: s12-s19.

16 Simon G, Coleman CC. Captopril-stimulated renal vein renin measurements in the diagnosis of atherosclerotic renovascular hypertension. Am 7 Hypertens 1994; 7: 1-6.

17 Pickering TG, Sos TA, James GD, Vaughan ED, Sealey JE, Laragh $\mathrm{H}$. Comparison of renal vein renin activity in hypertensive patients with stenosis of both renal arteries. 7 Hypertens $1985 ; 3$ (suppl 3): $s 291-93$

18 Hunt JC, Sheps SG, Harrison EG, Strong CG, Bernatz PE. Renal and renovascular hypertension. A reasoned approach to diagnosis and management. Arch Intern Med 1974; 133: 988-99.

19 Dean RH, Kieffer RW, Smith BM, et al. Renovascular hypertension, Anatomtic and renal funtion changes during drug therapy. Arch Surg 1981; 116: 1408-15.

20 Ramsay LE, Waller PC. Blood pressure response to percutaneous transluminal angioplasty for renovascular hypertension: an overview of published series. BMF 1990; 300: 569-72.

21 Weibull H, Bergqvist D, Bergentz SE, Jonsson K, Hulthen L, Manhem P. Percutaneous transluminal angioplasty versus reconstruction of atherosclerotic renal artery stenosis: prospective randomized study. 7 Vasc Surg 1993; 18: 841-52.

22 Plouin PF, Darne B, Chattelier G, et al. Restenosis after a first percutaneous transluminal renal angioplasty. Hypertension 1993; 21: 89-96.

23 Dorros G, Prince C, Mathiak L. Stenting of a renal artery stenosis achieved better relief of the obstructive lesion than balloon angioplasty. Cathet Cardiovasc Diagn 1993; 3: 191-98. 